Estructura del hogar como respuesta a los ajustes económicos: evidencia del México urbano de los ochenta* Rebeca, Wong** y Ruth E. Levine***

Este artículo presenta los resultados de un esfuerzo exploratorio para examinar los determinantes de la estructura de los hogares en algunas áreas urbanas de la República mexicana, el análisis se centra en aquellos hogares en los que viven mujeres con hijos pequeños.

En el trabajo se esboza un marco conceptual básico a la luz del cual se pueden analizar los determinantes de la estructura de los hogares en los países en vías de desarrollo; se describen las tendencias de las estructuras de los hogares y se presentan modelos de regresión muitivariada con el propósito de examinar los factores correlativos de la estructura de los hogares, con base en los datos de dos encuestas. Asimismo se presentan los resultados e implicaciones para otros aspectos demográficos de interés, tales como el empleo femenino y el cuidado de los hijos, y por último se incluyen algunas consideraciones sobre futuras líneas de investigación.

Para el análisis empírico se utilizan dos encuestas nacionales: la Encuesta Nacional Demográfica de 1982 y la Encuesta Nacional de Fecundidad y Salud de 1987, en un intento por detectar los cambios en los patrones de formación de los hogares. Estas dos encuestas cubren un periodo de deterioro económico de las áreas urbanas de México.

Los resultados sugieren que bajo las condiciones de deterioro que han aflorado en las áreas urbanas en México, como las existentes entre 1982 y 1987, la formación de hogares extendidos se incrementó, como una estrategia para apoyar la inserción de las mujeres en el mercado de trabajo asalariado, proveer de cuidado a los hijos pequeños, permitir a las mujeres más jóvenes el cuidado de sus hijos, y como una estrategia para apoyar el trabajo por cuenta propia dentro del hogar. También se encontró que tener mejores condiciones de vivienda estuvo asociado fuertemente con la extensión del hogar en 1987.

\title{
Introducción
}

El tipo de hogares que los individuos forman es una determinante importante de muchas consecuencias económicas, sociales y de-

\footnotetext{
* Investigación apoyada por la Fundación Rockefeller bajo el Programa de investigación sobre el estatus de la mujer y la fecundidad de la mujer en países en desarrollo.

** lohns Hopkins University, Baltimore, Maryland.

*** The Urban Institute, Washington, D.C.
} 
mográficas. El consumo, el ahorro, la participación de fuerza de trabajo femenina, la atención a los menores, y el cuidado de la salud son sólo algunos de los factores que están influidos por la organización de los hogares. Es bien sabido que las estructuras de los hogares varían de un sitio a otro, a través del tiempo y de las etapas del ciclo de vida del individuo. No obstante, aún sabemos poco acerca de aquellos factores que determinan las formas particulares de los hogares, de las diferencias de los determinantes en los distintos países, y sobre el efecto que las costumbres del lugar pueden tener en la organización de los hogares.

Este documento intenta sumarse al conocimiento de la formación de los hogares, empezando por examinar los determinantes de la estructura de los hogares en las áreas urbanas de México, en particular aquellos en los que viven mujeres con hijos pequeños. Seleccionamos este grupo de mujeres dado el interés de aplicar nuestro análisis a factores relacionados con la distribución del tiempo de la mujer dedicado, por ejemplo, a actividades de mercado y al cuidado de los menores. Nos enfocamos a las áreas urbanas, dado que en ellas se ha dado un crecimiento de las actividades que requieren fuerza de trabajo, sobre todo de la mujer. El análisis que presentamos constituye un esfuerzo de exploración, como parte de un antiguo interés en la participación de la mujer en la fuerza de trabajo.

La meta, finalmente, es incrementar el entendimiento de las relaciones entre ocupación de la mujer, fecundidad y empleo, y los factores que afectan su bienestar, incluyendo la estructura del hogar. Nuestro análisis empírico usa dos encuestas nacionales; en un intento por capturar los cambios en los patrones de comportamiento observamos los factores de las estructuras de hogares en dos momentos, 1982 y 1987, que cubren un periodo de deterioro económico en las áreas urbanas de México. El documento describe la variedad de estructuras del hogar en el área urbana de México, y la asociación de las formas de hogar con las principales características demográficas, sociales y económicas. Primero delineamos un marco conceptual básico para examinar los factores de las estructuras del hogar en países en desarrollo. En segundo lugar, describimos las tendencias de la estructura del hogar y otras variables importantes, usando la información de las dos encuestas. En tercero, usamos modelos de regresión multivariada para examinar los factores correlativos de la estructura del hogar, comparando las covariables en ambas encuestas.

Finalmente, discutimos nuestros resultados y las posibles implicaciones en otros comportamientos demográficos de interés, tales como el empleo de la mujer y el cuidado de los menores. 
También proporcionamos algunas consideraciones para investigaciones futuras.

\section{Marco conceptual}

Adoptamos la definición usual de hogar, como un grupo corresidente de individuos sin considerar su relación, ya sea por matrimonio, consanguinidad o afinidad. De manera general, un hogar está formado por individuos que comparten un lugar donde dormir y comer, y tienen un presupuesto común. Llamamos estructura del hogar al tipo de organización bajo la cual viven los individuos. Definimos como estructura extendida a aquella en la que uno o más miembros permanentes del hogar no pertenecen al núcleo familiar del jefe del hogar.

Para analizar la estructura del hogar como variable endógena, o resultante, adoptamos la teoría de producción del hogar, considerando al hogar como una unidad que combina el tiempo de los miembros con otros insumos para producir varios productos. Los miembros del hogar comparten algunos de estos productos, tales como las comidas y el cuidado de los menores, derivando una utilidad del consumo de los mismos.

Muchos autores de literatura económica han usado este marco para explicar la distribución del tiempo de los miembros del hogar, por ejemplo, la distribución del tiempo de la mujer en tareas domésticas o en actividades de mercado de trabajo. Este marco ha sido aplicado menos frecuentemente a modelos de formación del hogar en sí (Ermisch, 1988; Wolpin, 1984).

Ermisch (1988) señala que existen análisis económicos más detallados dirigidos a la formación de unidades domésticas al momento del matrimonio o el divorcio, a lo cual él llama "primer nivel de análisis". El "segundo nivel de análisis", o sea, los factores que determinan la estructura del hogar independientemente del matrimonio, ha recibido menos atención.

Para este último, se deben considerar aspectos de la preferencia por la privacidad y el costo de oportunidad de integrar a otros miembros (o miembros potenciales). Es necesario considerar, además, cómo los individuos se substituyen unos a otros en las actividades que requieren tiempo, y si hay economías (o deseconomías) de escala con un mayor número de miembros. Wolpin (1984) también señala "que los tipos prevalecientes de organización del hogar en cualquier sociedad deben [. . . ] resultar de un ambiente político, económico y tecnológico que haga que algunas formas organizacionales sean más eficientes que otras para alcanzar las 
metas del individuo dentro de la sociedad..." (Wolpin, 1984: 235).

Nos hace falta un modelo que especifique más claramente el comportamiento para adoptarlo en nuestros análisis centrados en los factores de la estructura del hogar. Sin embargo, hay algunos estudios de relevancia. De Vos y Palloni (1989) discuten el uso de los modelos formales para examinar la estructura del hogar, elaborando un útil resumen de los diversos enfoques. También presentan un marco para relacionar la disponibilidad de miembros en la familia, las reglas de la formación del hogar, y las restricciones demográficas con las estructuras observadas en el hogar.

Sin hacer una aplicación de los modelos de comportamiento, los autores presentan un marco en el cual las estructuras observadas del hogar son una función de las condiciones socioeconómicas, factores demográficos, normas de parentesco, reglas de formación y disolución del hogar y la disponibilidad de miembros de la familia.

En un estudio reciente de las decisiones individuales sobre la formación de los hogares, Mutchler y Burr (1991) examinan los diferentes tipos de hogar de la población de edad avanzada en Estados Unidos, sugiriendo que los recursos económicos influyen en su conformación, mientras que las condiciones de salud explican la institucionalización. En lo que respecta a Latinoamérica, varios estudios descriptivos han documentado la composición demográfica de los hogares y unidades domésticas, sin que proporcionen, desde nuestro punto de vista, un modelo de comportamiento para las estructuras del hogar. DeVos (1985) describe la estructura de los hogares en varios países de Latinoamérica. Tienda y Ortega (1980) exploran en las zonas rural y urbana de Perú si el tipo de jefe del hogar afecta la propensión a tener uno o más parientes viviendo en éstos. Los autores concluyen que los hogares extendidos ayudan a compensar la ausencia de un cónyuge en hogares encabezados por una mujer.

Zúñiga et al. (1986) presentan una descripción del México rural, donde los hogares tienden a ser nucleares. Comparando con los medios rurales, los hogares que no son nucleares (o sea, hogares extendidos) son más comunes en las áreas urbanas. Los autores concluyen que los hogares extendidos parecen ser un arreglo para compensar la alta migración o la alta mortalidad en el México rural.

Cuéllar (1990) enfatiza la etapa de desarrollo económico en la formación de los hogares extendidos. Asimismo, demuestra que en las áreas rurales de México, la variación en el porcentaje de los hogares extendidos es grande (entre 8 y $40 \%$ ), a pesar de que pre- 
dominan los hogares nucleares. El autor también menciona que entre los hogares rurales pobres, aquellos con mayor número de miembros viven en mejores condiciones que aquellos con pocos, tal vez debido a una estrategia de supervivencia de agregación de individuos.

Para nuestro marco conceptual, suponemos que todos los individuos que forman un hogar son personas que pueden tomar decisiones, a pesar de que ciertas decisiones se toman en pareja, otras involucran a la familia, y otras más son hechas por un solo individuo (el jefe del hogar, por ejemplo). Una de las decisiones que una pareja puede tomar es formar un hogar con individuos que no pertenecen directamente a la familia o al grupo del núcleo. La pareja puede optar por vivir sola, con los padres de lá esposa, o con otros parientes o adultos no emparentados con ellos. Desde una perspectiva económica, la alternativa que se tome será maximizar los productos de su elección, de manera más eficiente que lo que sería posible en otro tipo de hogar. Debido a que la estructura seleccionada es un resultado de decisiones de producción y consumo, es difícil separar los efectos en la estructura del hogar dadas ciertas economías tecnológicas de escala (la unidad como productor), de aquellas surgidas por las preferencias (la unidad como consumidor). Sin embargo, usando esta teoría básica, podemos proponer los factores explicativos a incluirse en un modelo de estructuras del hogar. También podemos especular acerca de los efectos esperados de ciertas variables socioeconómicas sobre las estructuras del hogar. Por ejemplo, un mayor ingreso tendería a producir estructuras del hogar que facilitarian el consumo de bienes comunes.

Si la norma social para la pareja es vivir en un hogar de tipo nuclear, entonces los hogares tenderán hacia esta estructura cuando el ingreso aumente. Si por otro lado, la norma social es vivir con la familia de uno de los cónyuges cuando el matrimonio empieza, entonces mayores ingresos estarán asociados con estos tipos de hogares. Si la privacidad es vista como un bien normal, entonces ceteris paribus, la posibilidad de formar un hogar de núcleo separado crecerá cuando aumente el ingreso. Por otro lado, el efecto del aumento de precios será opuesto al efecto del ingreso. Un incremento en el costo de la vivienda (produciendo una disminución en el ingreso) tenderá a disminuir la posibilidad de formar hogares de núcleos separados (Ermisch, 1988).

Optar por un hogar extendido en lugar de uno de tipo nuclear dependerá de los beneficios relativos de tener miembros fuera del núcleo familiar, comparado con los costos que los mismos provocan, dadas las restricciones de cada individuo. En consecuencia, 
la estructura de hogar observada depende de: las preferencias de los miembros, en especial su gusto relativo por la privacidad en relación con el consumo de bienes (medidos, por ejemplo, por la edad, la cultura, etc.); su habilidad relativa para desempeñar tareas del hogar (medidas por composición por sexo, número de hijos, etc.); capacidad de ingresos en términos reales (medidos por la educación de los miembros, salarios, región de residencia, y el in greso no-monetario de la unidad, medido por el tipo de vivienda, activo fijo, etcétera).

A causa de la poca literatura que presenta evidencia empírica sobre los determinantes de la estructura del hogar, y debido a que dichos determinantes dependen del contexto específico, tenemos dificultades para predecir la dirección de las asociaciones entre la estructura del hogar y sus principales variables explicativas. En nuestro modelo de interrelaciones de la estructura del hogar (medido como hogar extendido, o nuclear), esperamos que, manteniendo las demás variables constantes:

- Un ingreso más alto per cápita (o por persona) se asociará a una estructura nuclear. Si suponemos que en las zonas urbanas de México se prefieren las estructuras con mayor privacidad, ingresos más altos estarán asociados con estructuras nucleares. De modo similar, se asociarán las mejores condiciones de vivienda con los hogares nucleares. Sin embargo, un mayor ingreso y mejor vivienda se pueden asociar con la habilidad de mantener a un mayor número de miembros, dadas las economías de escala que se tienen en los hogares extendidos. Por lo tanto, el efecto del tipo de vivienda (una medida de ingrese no-monetaria) y del ingreso puede ser ambiguo.

- Se asociará con las estructuras de tipo nuclear una capacidad de ingresos más alta de la mujer (medida por su educación). Por otro lado, las mujeres con mejor preparación tenderán a tener un costo de oportunidad del tiempo más alto, dependiendo de las oportunidades disponibles en el mercado de trabajo. Las mujeres con un costo de oportunidad más alto tenderán hacia hogares extendidos que les permitan participar en el mercado de trabajo, especialmente si tienen niños. Por lo tanto el efecto de la educación puede ser ambiguo en la ausencia de un control en la participación en el mercado de trabajo.

- El tipo de hogar seleccionado se basará en las preferencias, las cuales cambian con el ciclo de la vida. Esperamos que las edades más jóvenes estén asociadas con las estructuras nucleares, puesto que suponemos que la privacidad es un factor deseado especialmente por las parejas jóvenes. Por otro lado, 
otro aspecto que cambia con el ciclo de la vida es la habilidad de formar hogares separados. Mujeres más jóvenes pueden encontrar que uniéndose con otros miembros (fuera del núcleo) tendrán como resultado economías de escala, especialmente cuando no se han acumulado ahorros, lo cual es más común en el caso de personas jóvenes. Por lo tanto, es más factible encontrar mujeres jóvenes en los hogares extendidos que mujeres mayores. Esto implica que el efecto de la edad puede ser ambiguo.

- El estar casado o en unión consensual se asociará con las estructuras nucleares. Dado que nuestra muestra incluye a todas las mujeres con hijos pequeños, las madres solteras tal vez encontrarán más deseable vivir en hogares extendidos, dadas las economías de escala y por las actitudes culturales que podrían criticar a una mujer que cría sola a sus hijos.

- El número de hijos pequeños, o el tener un hijo pequeño, se asociará positivamente con las estructuras extendidas, dado que este tipo de estructura puede facilitar el cuidado de los niños a bajo costo.

- Las mujeres con antecedentes rurales tenderán a vivir en estructuras extendidas, puesto que es más factible que tengan parientes procedentes de áreas rurales dispuestos a formar hogares extendidos temporalmente, como una transición a establecerse en la zona urbana.

- Las mujeres que viven en las áreas metropolitanas serán más dadas a vivir en hogares extendidos, puesto que el costo de la vivienda es más alto que en otras zonas urbanas más pequenas. Las áreas metropolitanas pueden ofrecer más y mejores oportunidades para que la mujer participe en ocupaciones pagadas, y esto a su vez puede hacer que los hogares extendidos sean más atractivos para las mujeres que viven en áreas metropolitanas.

\section{Información y métodos}

Usamos dos fuentes de información para México: La Encuesta Nacional Demográfica de 1982 y La Encuesta Nacional de Fecundidad y Salud de 1987. Ambas encuestas incluyen historias de matrimonio y reproducción para las mujeres de entre 15 y 49 años de edad, su nivel de educación, el estatus ocupacional actual e información general de cada miembro del hogar (edad, sexo, educación, empleo, relación con el jefe del hogar). Del total de muestras nacionales, las submuestras para nuestros análisis incluyen a las mujeres que viven en las áreas urbanas (definidas como comuni- 
CUADRO 1

Descripción estadística de las principales variables ${ }^{1}$

\begin{tabular}{lcc}
\hline & \multicolumn{2}{c}{ Porcentaje } \\
\cline { 2 - 3 } Variable & 1982 & $1987^{2}$ \\
\hline Hogares extendidos & 36.7 & 37.1 \\
Vertical & 56.8 & 56.6 \\
Lateral & 27.5 & $33.0^{* *}$ \\
Ambos vertical y lateral & 15.8 & $10.3^{* * *}$ \\
Escolaridad de la mujer & & \\
$>$ de 6 años & 38.0 & $45.7^{* * *}$ \\
Edad de la mujer $\geq 27$ años & 54.8 & $56.2^{* * *}$ \\
Edad del último hijo $\geq 24$ meses & 43.2 & $48.1^{* *}$ \\
Empleada & 19.1 & $28.8^{* *}$ \\
Casada/en unión & 89.8 & $92.1^{* * *}$ \\
Ciudad de México & 35.8 & $38.8^{*}$ \\
Monterrey & 6.3 & 5.8 \\
Guadalajara & 6.7 & $6.2^{*}$ \\
Compañero que trabaja & & \\
por cuenta propia & 21.9 & 19.7 \\
Servicios de la vivienda & 58.9 & 61.3 \\
Antecedentes rurales & 33.7 & $37.8^{* * *}$ \\
Núm. total & 2939 & 1914 \\
\hline
\end{tabular}

${ }^{1}$ Datos ponderados.

${ }^{2}$ Diferencias significativas entre los porcentajes de 1982 y 1987 a:

$*$ Nivel .05

** Nivel .02

*** Nivel .01

dades con más de 20000 habitantes) y quienes han tenido por lo menos un hijo dentro de los cinco años anteriores a cada encuesta.

El apéndice A presenta las definiciones utilizadas en ambas encuestas. Hicimos todo lo posible para definir de manera similar las variables que nos interesan. Los cuadros 1 y 2 presentan resúmenes descriptivos de las principales variables de ambas submuestras. En 1982 se tiene la cifra de 2939 mujeres y 1914 en 1987. Todos nuestros análisis incluyen los datos ponderados, para ajustar las diferencias que existen en los diseños del muestreo de estas encuestas.

Para describir más ampliamente los hogares que son extendidos, construimos una medida del tipo de extensión. Definimos una extensión vertical como una extensión en la que los miembros adicionales (es decir, fuera del grupo nuclear) están relacionados con el jefe del hogar y pertenecen a una generación distinta (por ejemplo, nieto, madre, suegra). Llamamos hogares extendidos con extensión lateral a aquellos en los que los miembros fuera del nú- 
cleo pertenecen a la misma generación del jefe (por ejemplo, hermana, prima, cuñada). También hay hogares con extensión lateral y vertical, si los miembros fuera del núcleo son algunos de la misma y otros de otra generación que el jefe del hogar. Como se puede ver en el cuadro 1 , en las dos encuestas, aproximadamente $37 \%$ de las mujeres viven en hogares extendidos. La distribución según el tipo de hogar extendido difiere ligeramente. En la información que presentan las encuestas de 1982 y 1987 , resulta que $57 \%$ de las mujeres que viven en hogares extendidos se encontraban dentro de una estructura vertical. La distribución de los otros dos tipos de hogares extendidos difiere entre un año y otro. En 1982, 27\% de las mujeres estaban viviendo en hogares extendidos con estructura de forma lateral, y $16 \%$ en ambas estructuras lateral y vertical. En $1987,33 \%$ estaban en estructura lateral y $10 \%$ en estructuras vertical y lateral.

CUADRO 2

Descripción estadística de las principales variables por tipo de estructura del hogar ${ }^{1}$

\begin{tabular}{|c|c|c|c|c|c|}
\hline \multirow[b]{3}{*}{ Características } & \multicolumn{5}{|c|}{$\begin{array}{l}\text { Porcentaje en hogares extendidos o } \\
\text { nucleares con características }\end{array}$} \\
\hline & \multicolumn{2}{|c|}{1982} & \multicolumn{3}{|c|}{1987} \\
\hline & Nucleares & Extendidos $^{2}$ & Nucleares & Extend & idos $^{2}$ \\
\hline Mujeres con escolaridad & & & & & \\
\hline $\begin{array}{l}>6 \text { años } \\
\text { Edad de la mujer }\end{array}$ & 35.4 & $42.5^{* * *}$ & 42.4 & 51.3 & $* * *$ \\
\hline $\begin{array}{l}\geq 27 \text { años } \\
\text { Edad del último hiio }\end{array}$ & 58.7 & $48.1^{* * *}$ & 61.4 & 47.4 & ${ }^{* * *}$ \\
\hline$\geq 24$ meses & 43.5 & 42.8 & 50.2 & 44.5 & ** \\
\hline Empleada & 15.3 & $25.8^{* * *}$ & 23.1 & 38.3 & $* * *$ \\
\hline Casada/en unión & 96.7 & $77.9^{* * *}$ & 98.6 & 81.1 & $* * *$ \\
\hline Ciudad de México & 36.6 & 34.5 & 40.0 & 36.9 & \\
\hline Monterrey & 6.4 & 6.1 & 6.2 & 5.1 & \\
\hline Guadalajara & 6.7 & 6.8 & 6.2 & 6.1 & \\
\hline Compañero trabajando & & & & & \\
\hline por cuenta propia & 19.5 & $26.2 * * *$ & 16.7 & 24.9 & *** \\
\hline Servicios de la vivienda & $\mathbf{5 7 . 0}$ & $62.4 * * *$ & 59.8 & 63.8 & \\
\hline Antecedentes rurales & 34.9 & 31.8 & 36.6 & 39.7 & \\
\hline Núm. total & 1860 & 1079 & 1203 & 711 & \\
\hline
\end{tabular}

${ }^{1}$ Datos ponderados.

2 Diferencias significativas entre los porcentajes de 1982 y 1987 a:

* Nivel .05

** Nivel .02

*** Nivel .01 
El cuadro 1 también revela que en 1987 las madres con hijos pequeños tenían mejor educación ( $46 \%$ tenían más de 6 años de escolaridad, comparado a un $38 \%$ de 1982). El último hijo de estas mujeres, nacido antes de 1987, era sólo un poco mayor que los de 1982 y eran más o menos de la misma edad que aquellos registrados en la muestra de 1982 . Una mayor proporción de madres era asalariada en 1987 (29\% comparado con un 19\% de 1982). Cerca de $\mathbf{9 0 \%}$ de las madres entre 1982 y 1987 eran casadas o vivían en unión consensual, con un porcentaje ligeramente mayor en 1987. Una proporción mayor tenía antecedentes rurales en relación con la muestra de 1982 (38\% comparado con un 34\%). Aproximadamente $40 \%$ de las madres, en ambas encuestas, deseaban tener otro hijo. Las condiciones de vivienda, en ambas submuestras, eran similares con respecto a los servicios: cerca de $60 \%$ de las madres vivian en hogares que contaban con tres servicios (electricidad, agua y drenaje). En los dos años, cerca de $20 \%$ de las madres tenía un compañero o jefe de hogar que trabajaba por cuenta propia. La distribución geográfica en las tres áreas metropolitanas de México fue muy similar, a excepción de una proporción ligeramente más alta en la ciudad de México en 1987.

El cuadro 2 presenta estadísticas descriptivas de las principales variables de interés, por tipo de estructura de hogar (nuclear o extendido). Las estadísticas muestran que los hogares extendidos se asocian con mujeres mejor educadas, más jóvenes, empleadas y madres solteras en 1982 y 1987 . Vivir en un hogar extendido, durante estos años, también se relaciona con el hecho de que tenían un compañero trabajando por cuenta propia. El tener un niño pequeño está asociado con el hogar extendido sólo en 1987, y el vivir en mejor vivienda sólo en 1982.

Para examinar las covariables de la estructura de hogar (extendido o no) como una variable dependiente, estimamos ecuaciones de regresión logística con una serie de variables explicativas que representan los factores hipotéticos como determinantes en nuestro marco conceptual. Los modelos multivariados excluyen el empleo de la mujer como variable explicatoria, dada la posible endogeneización del empleo con la estructura del hogar. Por lo tanto, aquí estimamos una forma reducida del modelo, dejando la estimación de la forma estructural como tema de una futura investigación.

El cuadro 3 presenta los resultados de los modelos de regresión para 1982 y 1987. Los modelos multivariados confirman muchas de las asociaciones encontradas en los análisis descriptivos. Manteniendo las demás variables constantes en ambos años, encontramos que los hogares extendidos se relacionan con las 
CUADRO 3

Coeficientes de regresión logística ${ }^{1}$ variable dependiente: hogar extendido $(s i=1)$

\begin{tabular}{|c|c|c|c|c|}
\hline \multirow[b]{2}{*}{ Variable } & \multicolumn{4}{|c|}{ Coeficiente de regresión } \\
\hline & \multicolumn{2}{|l|}{$1982^{2}$} & \multicolumn{2}{|l|}{$1987^{2}$} \\
\hline Intercepción & 4.14 & $* * *$ & 7.16 & $* * *$ \\
\hline Escolaridad de la mujer & .025 & $* *$ & .037 & $* *$ \\
\hline Edad de la mujer & -.192 & $* * *$ & -.340 & $* * *$ \\
\hline Edad al cuadrado de la mujer & .002 & $* * *$ & .004 & $* * *$ \\
\hline Casada/en unión & -2.09 & $* * *$ & -2.853 & $* * *$ \\
\hline Ciudad de México & -.087 & & -.065 & \\
\hline Monterrey & .006 & & -.228 & \\
\hline Guadalajara & -.051 & & -.161 & \\
\hline \multicolumn{5}{|l|}{ Compañero trabajando por } \\
\hline cuenta propia & .444 & $* * *$ & .683 & $* * *$ \\
\hline Servicios de la vivienda & .217 & $* *$ & .322 & $* * *$ \\
\hline Edad del último hijo & .000 & & -.007 & $* *$ \\
\hline Antecedentes rurales & .055 & & .272 & ** \\
\hline Núm. total & 2939 & & 1914 & \\
\hline$-2 \log L$. & 3534 & & 2219 & \\
\hline$\%$ extendido & 36.7 & $\%$ & 37.1 & $\%$ \\
\hline
\end{tabular}

${ }^{1}$ Datos ponderados.

${ }^{2}$ Coeficientes de regresión significativamente diferentes de cero a:

* Nivel .01

** Nivel .05

*** Nivel .10

mujeres que cuentan con más años de educación, son más jóvenes y están fuera de una unión, con compañeros que trabajan por cuenta propia y con mejores viviendas. Encontramos diferencias notables entre 1982 y 1987: la edad del último hijo y los antecedentes de la mujer no son factores significativos para la explicación de los hogares extendidos en 1982 pero sí lo son para 1987. Para este año, el vivir en hogares extendidos se asocia con antecedentes rurales y con el hecho de que el último hijo nacido sea muy joven. Para ambos años, la región de residencia no es una variable significativa para su explicación.

Los resultados de los análisis multivariados corresponden, en gran parte, a los efectos que esperábamos tal como los delineamos en el marco conceptual. Formulamos la hipótesis de que el efecto de mejores viviendas y la edad de la mujer serían ambiguos. Obtuvimos un efecto negativo de la edad y uno positivo de mejores viviendas en la probabilidad de vivir en hogares extendidos. Un resultado inesperado fue el efecto positivo de la educación en hogares extendidos. Especulamos que el efecto de la educación en la probabilidad de formar un hogar extendido representa el efecto 
CUADRO 4-A

Porcentaje de mujeres con determinadas características, que tienen más de 6 años de educación

\begin{tabular}{lccc}
\hline & $1982(\%)$ & $1987(\%)$ \\
\hline Todas las mujeres & 38.0 & $45.77^{* * *}$ \\
Hogares nucleares & 35.4 & $42.44^{* *}$ \\
Hogares extendidos & 42.5 & $51.44^{* *}$ \\
Empleadas & 64.6 & $51.7^{* * *}$ \\
No-empleadas & 31.7 & $43.3^{* * *}$ \\
\hline
\end{tabular}

Nota: datos ponderados. Diferencias significativas en los porcentajes entre 1982 y 1987 a:

$*$ Nivel .05

*** Nivel .02

*** Nivel .01

del empleo de la mujer. Exploramos este aspecto examinando más a fondo la relación entre el empleo de la mujer, su educación y la estructura del hogar.

En los cuadros 4-A y 4-B se presenta el porcentaje de mujeres con más de seis años de escolaridad de acuerdo con el estatus ocupacional y la estructura de hogar, para ambas encuestas. En el cuadro 4-A se muestra que, en ambos años, las mujeres en hogares extendidos tienen una mejor preparación que aquellas que viven en hogares nucleares; también encontramos que las mujeres que trabajan tienen más años de escolaridad que aquellas que no trabajan. Todas las mujeres tienen mayor educación en la muestra de 1987 que en la de 1982. Este resultado surge tanto para las mujeres viviendo en hogares extendidos como para hogares nucleares. Sin embargo, la relación no se conserva al separar a las mujeres por estatus ocupacional. Entre las mujeres que se encuentran emplea-

CUADRO 4-B

Porcentaje de mujeres con determinadas características, que tienen más de 6 años de educación para 1982 y 1987

\begin{tabular}{lccc}
\hline & Nuclear (\%) & \multicolumn{3}{c}{ Extendido (\%) } \\
\hline 1982 - Empleada & 69.7 & 59.3 & $* * *$ \\
1987 - Empleada & 53.4 & 50.0 & \\
& & & \\
1982 - No-empleada & 29.2 & 36.6 & $* * *$ \\
1987 - No-empleada & 39.1 & 52.3 & $* * *$ \\
\hline
\end{tabular}

Nota: datos ponderados. Diferencias significativas en los porcentajes entre hogares extendidos y nucleares a:

* Nivel .05

** Nivel .02

*** Nivel .01 
das, las de 1982 contaban con una mejor educación. Este cuadro también muestra que la diferencia educacional entre las mujeres empleadas y las no-empleadas es mayor en 1982 que en 1987. El cuadro 4-B muestra que entre las mujeres empleadas, la educación es mayor entre las mujeres viviendo en hogares nucleares que en los extendidos. Lo contrario sucede para las mujeres noempleadas: aquellas que tienen hogares extendidos cuentan con mayor educación. Este resultado general no se cumple para las mujeres empleadas en 1987, donde no encontramos diferencias en la educación a través de las estructuras extendidas y nucleares. El cuadro 4-B muestra también que, como indican los resultados del cuadro 4-A, la brecha educacional entre las mujeres empleadas y no empleadas se cerró entre 1982 y 1987, pero especialmente para aquellas que vivían en hogares extendidos, donde $50 \%$ comparado con $52 \%$ de mujeres tienen más de seis años de estudios (véase diagrama l). Este grupo de resultados confirma la hipótesis de que la relación entre la educación y la estructura del hogar estaba cubriendo el efecto del empleo. También confirmamos que, para separar estos efectos, el empleo de la mujer debe ser modelado como endógeno de la estructura del hogar.

\section{Discusión y conclusiones}

Hemos presentado los resultados de un análisis exploratorio, conducente a un modelo de determinantes de la estructura del hogar en el México urbano. Hicimos nuestros análisis usando como muestras a mujeres con hijos pequeños en dos encuestas nacionales de 1982 y 1987, cubriendo un periodo de deterioro económico en el México urbano. Encontramos que la distribución de las madres en hogares nucleares o extendidos permanecieron relativamente estables entre 1982 y 1987.

Nuestro marco conceptual nos proporcionó un perfil del modelo de determinantes de la estructura del hogar. Encontramos que nuestro análisis empírico confirmó la mayoría de los efectos enunciados en nuestras hipótesis para las variables económicas y demográficas sobre la probabilidad de formar hogares extendidos. En nuestras submuestras de madres con hijos pequeños, encontramos que para las madres jóvenes y no-unidas, es más probable vivir en hogares extendidos. Cuando se controla la edad y el estado civil, las mujeres que trabajan por cuenta propia y están en mejores condiciones económicas tienen posibilidades de pertenecer también a un hogar extendido. Igualmente, fue un resultado importante que los hogares extendidos fueran más comunes entre 
DIAGRAMA 1

Educación de la mujer por estructura del hogar y el empleo

\% de mujeres con más de 6 años de educación

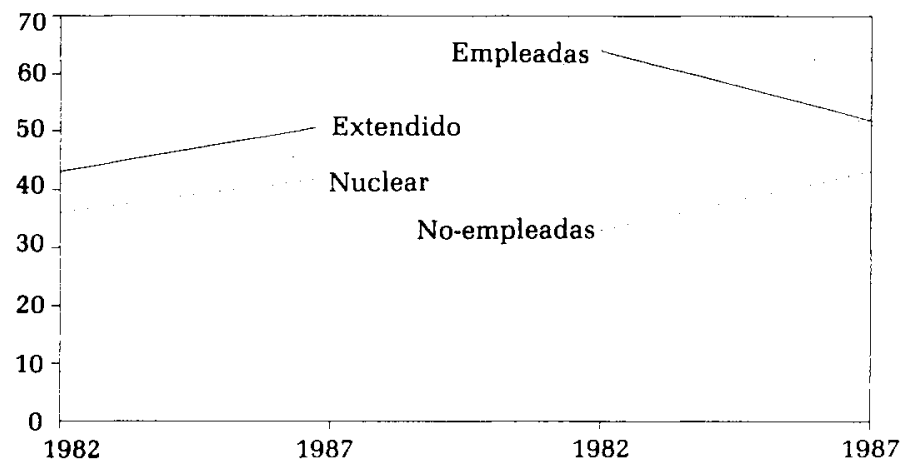

Fuente: Cuadro 4-A

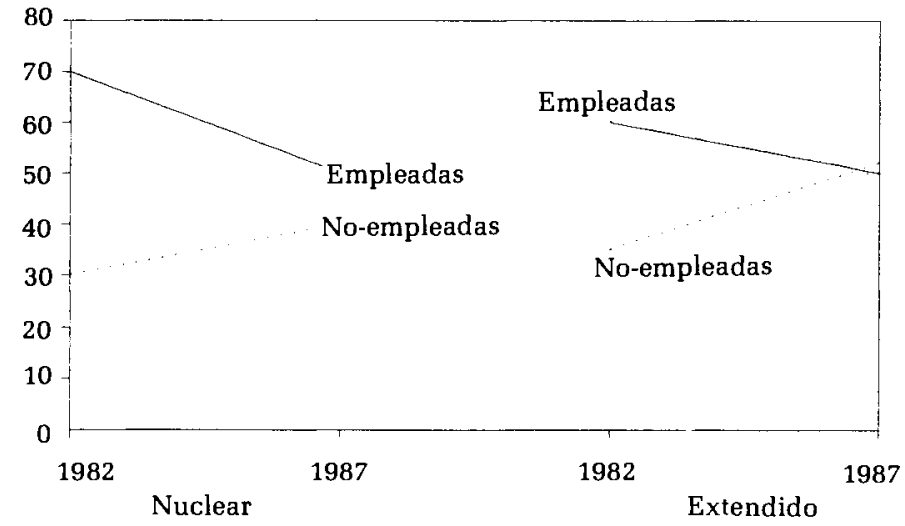

Fuente: Cuadro 4-B. 
las mujeres con mayor educación. El efecto de la educación fue en gran parte un resultado del empleo, dado que una mujer con mayor educación estaba en mejores condiciones de encontrar un trabajo asalariado. Un examen más a fondo de este aspecto en un análisis bivariado nos condujo a concluir que la extensión del hogar se asociaba con el empleo, cerrando la brecha educacional entre mujeres empleadas y no-empleadas.

Nuestros resultados apoyan la idea de que los hogares extendidos se formaron en el México urbano para resolver las demandas de tiempo de los miembros del hogar. No logramos encontrar apoyo para la idea de que la estructura de los hogares es resultado de la preferencia por la privacidad: los efectos de las hipótesis que apoyarían la preferencia por ésta no concordaron con las muestras de este análisis. Las condiciones en el proceso de modernización, en donde un mejor ingreso y una mayor educación se asociaban a la vida en un hogar nuclear, no concuerda con el México urbano, por lo menos para este grupo de mujeres con hijos pequeños.

Examinamos dos grupos de datos para estudiar las covariables de las estructuras del hogar a través del tiempo. Encontramos que los efectos de la educación de la mujer, su edad, que el compañero trabajara por cuenta propia, y la calidad de la vivienda fueron mayores en 1987 que en 1982. El efecto de tener un hijo pequeño y antecedentes rurales se volvieron estadísticamente significativos en 1987. Estos resultados sugieren que bajo las condiciones de deterioro económico de las áreas urbanas de México (como prevalecieron entre 1982 y 1987), formar hogares extendidos fue creciendo como estrategia para apoyar la participación de las mujeres en un empleo asalariado, para proveer de cuidado a los hijos pequeños, para permitir a las mujeres más jóvenes cuidar a su(s) hijo(s), y como estrategia para apoyar el trabajo por cuenta propia. También encontramos que el tener mejor vivienda se volvió más fuertemente asociado con la extensión del hogar en 1987. Este resultado, combinado con una mayor probabilidad de tener un hogar extendido entre las mujeres de antecedentes rurales, apoya la noción de que al empeorar las condiciones económicas del México urbano, los hogares con mejores condiciones económicas tenían una mayor probabilidad de formar extensiones para proveer de un apoyo a los otros miembros, tal vez de áreas rurales.

\section{Bibliografía}

Conapo (1982), "Encuesta Nacional Demográfica, Documento Conceptual", en National Demographic Survey, Conceptual Document, México. 
Cuéllar, O. (1990), "Las familias campesinas numerosas viven menos mal" (Peasant Families that are Numerous Fare Less Worse Off), en Demos. Carta Demográfica sobre México, núm. 3.

De Vos S. (1985), "Latin American Households in Comparative Perspective", Madison, Universidad de Wisconsin, Documento de Trabajo, CDE, Centro de Demografía y Ecología.

y A. Palloni (1989), "Formal Models and Methods for the Analysis of Kinship and Household Organization", en Population Index, núm. 55 (2), pp. 174-198.

Ermisch, J. (1988), "An Economic Perspective on Household Modelling", en N. Keilman, A. Kuijstein y A. Vossen (eds.), Modelling Household Formation and Dissolution, Oxford, Clarendon Press.

García, B. y O. de Oliveira (1979), "Una caracterización sociodemográfica de las unidades domésticas en la ciudad de México", en Demografía y Economía, vol. xiII, pp. 1-17.

Mutchler, J. y J. Burr (1991), "A longitudinal analysis of household and nonhousehold living arrangements in later life", en Demography, vol. 28, núm. 3, agosto.

Safilios-Rothschild, C. (1989), "Theoretical aspects of the family systems of the less and more industrialized countries: are all family systems converging?", en IUSSP Meetings, pp. 119-127.

Shapiro, D. y F.L. Mott (1992), "Long-Term employment and earnings of women in relation to employment behaviour surrounding the first birth", paper presented at the North American Economics and Finance Association, Nuevo Orleans, Allied Social Sciense Associations Annual Meetings, enero.

Tienda, M. y S. Ortega (1980), "Female-Headed Households and Extended Family Formation in Rural and Urban Peru" (manuscrito sin publicar).

y J. Glass (1985), "Household structure and labor force participation of black, hispanic, and white mothers", en Demography, 22(3), pp. 381-394.

Wolf, D. A. y B. J. Soldo (1988), "Household composition choices of older unmarried women", en Demography, 25(3), pp. 387-403.

Wolpin, K. (1984), "The household structure of U.S. immigrants and natives", en Research in Population Economics, vol. 5, Jai Press, pp. 235-250.

Zúñiga, E., D. Hernández, C. Menkes, y C. Santos (1986), Trabajo familiar, conducta reproductiva y estratificación social (Family labor, reproductive behavior and social stratification), México, Instituto $\mathrm{Me}$ xicano del Seguro Social. 
APÉNDICE A

Definición de las variables

\begin{tabular}{|c|c|}
\hline Variable & Definición \\
\hline $\begin{array}{l}\text { Hogar extendido } \\
(s i=1)\end{array}$ & $\begin{array}{l}\text { Si uno o más de los miembros permanentes } \\
\text { del hogar no forman parte del núcleo } \\
\text { familiar del jefe del hogar. }\end{array}$ \\
\hline $\begin{array}{l}\text { Hogar extendido } \\
\text { vertical }(s i=1)\end{array}$ & $\begin{array}{l}\text { Si los miembros que no son del núcleo } \\
\text { pertenecen a una generación diferente a la } \\
\text { del jefe del hogar. }\end{array}$ \\
\hline $\begin{array}{l}\text { Hogar extendido } \\
\text { lateral }(\mathrm{si}=1)\end{array}$ & $\begin{array}{l}\text { Si los miembros que no son del núcleo del } \\
\text { hogar pertenecen a la misma generación que } \\
\text { la del jefe del hogar. }\end{array}$ \\
\hline $\begin{array}{l}\text { Hogar extendido } \\
\text { ambos vertical y } \\
\text { lateral }(\mathrm{si}=1)\end{array}$ & $\begin{array}{l}\text { Si los miembros que no son del núcleo } \\
\text { pertenecen a la misma y a diferentes } \\
\text { generaciones que la del jefe del hogar. }\end{array}$ \\
\hline $\begin{array}{l}\text { Escolaridad de la mujer } \\
>6 \text { años }(\mathrm{si}=1)\end{array}$ & $\begin{array}{l}\text { Si la mujer tiene más de } 6 \text { años de } \\
\text { educación formal. }\end{array}$ \\
\hline Escolaridad de la mujer (años) & Años de educación formal de la mujer. \\
\hline $\begin{array}{l}\text { Edad de la mujer } \\
\geq 27 \text { años }(\mathrm{si}=1)\end{array}$ & Si la mujer tiene 27 años o más. \\
\hline Edad de la mujer (años) & Edad de la mujer en años. \\
\hline $\begin{array}{l}\text { Edad del último hijo } \\
\geq 24 \text { meses }(s i=1)\end{array}$ & $\begin{array}{l}\text { Si el último hijo que tuvo la } \\
\text { mujer tiene } 2 \text { años o más. }\end{array}$ \\
\hline Edad del último hijo (meses) & La edad del último hijo en meses. \\
\hline Empleada $(s i=1)$ & Si la mujer trabaja por un salario. \\
\hline Casada/en unión(si = 1) & $\begin{array}{l}\text { Si la mujer es casada o vive en unión } \\
\text { consensual. }\end{array}$ \\
\hline $\begin{array}{l}\text { Ciudad de México } \\
(\mathrm{si}=1)\end{array}$ & $\begin{array}{l}\text { Si la mujer radica en el área } \\
\text { metropolitana de la Cd. de México. }\end{array}$ \\
\hline $\begin{array}{l}\text { Monterrey } \\
(\mathbf{s i}=1)\end{array}$ & $\begin{array}{l}\text { Si la mujer radica en el área metropolitana } \\
\text { de Monterrey, Nuevo León. }\end{array}$ \\
\hline $\begin{array}{l}\text { Guadalajara } \\
(s i=1)\end{array}$ & $\begin{array}{l}\text { Si la mujer radica en el área metropolitana } \\
\text { de Guadalajara, Jalisco. }\end{array}$ \\
\hline $\begin{array}{l}\text { Compañero que trabaja } \\
\text { por cuenta propia } \\
(\mathrm{si}=1)\end{array}$ & $\begin{array}{l}\text { Si el compañero de la mujer o el jefe del } \\
\text { hogar trabaja de forma independiente } \\
\text { (es decir, por cuenta propia). }\end{array}$ \\
\hline $\begin{array}{l}\text { Servicios de la vivienda } \\
(s i=1)\end{array}$ & $\begin{array}{l}\text { Si la vivienda donde la mujer vive tiene los } \\
\text { tres servicios: agua, electricidad y drenaje. }\end{array}$ \\
\hline $\begin{array}{l}\text { Antecedentes rurales } \\
(\mathrm{si}=1)\end{array}$ & $\begin{array}{l}\text { Si la mujer nació en un rancho, } \\
\text { pueblo o municipio. }\end{array}$ \\
\hline
\end{tabular}


